\title{
Epidemic spreading on complex networks with general degree and weight distributions
}

\author{
Wei Wang, ${ }^{1}$ Ming Tang,, , 2, $*$ Hai-Feng Zhang, ${ }^{3}$ Hui Gao, ${ }^{1}$ Younghae Do, ${ }^{4}$ and Zong-Hua Liu ${ }^{5}$ \\ ${ }^{1}$ Web Sciences Center, University of Electronic Science and Technology of China, Chengdu 610054, China \\ ${ }^{2}$ Center for Atmospheric Remote Sensing(CARE), Kyungpook National University, Daegu, 702-701, South Korea \\ ${ }^{3}$ School of Mathematical Science, Anhui University, Hefei 230039, China \\ ${ }^{4}$ Department of Mathematics, Kyungpook National University, Daegu 702-701, South Korea \\ ${ }^{5}$ Department of Physics, East China Normal University, Shanghai 200062, China
}

(Dated: June 15, 2021)

\begin{abstract}
The spread of disease on complex networks has attracted widely attention in the physics community. Recent works have demonstrated that heterogeneous degree and weight distributions have a significant influence on the epidemic dynamics. In this study, a novel edge-weight based compartmental approach is developed to estimate the epidemic threshold and epidemic size (final infected density) on networks with general degree and weight distributions, and a remarkable agreement with numerics is obtained. Even in complex network with the strong heterogeneous degree and weight distributions, this approach is worked. We then propose an edge-weight based removal strategy with different biases, and find that such a strategy can effectively control the spread of epidemic when the highly weighted edges are preferentially removed, especially when the weight distribution of a network is extremely heterogenous. The theoretical results from the suggested method can accurately predict the above removal effectiveness.
\end{abstract}

PACS numbers: 89.75.Hc, 87.19.X-, 87.23.Ge

\section{INTRODUCTION}

In most real-world networks, edges connecting two nodes are often associated with weights that differentiate them in terms of their strength, intensity, or capacity [1]. For example, in scientific collaboration networks, the weight of each edge can stand for the number of papers that two authors have coauthored [2, 3]; in communication networks, it can represent the total duration of calls between two users over a period of time [4]; and in brain networks, it can be viewed as the times of memories reinforced between neurons [5, 6]; also, it can account for the number of passengers between two airports in aviation networks [7].

A large number of empirical studies have verified that the degree and weight distributions of many weighted networks are greatly heterogeneous [8] (e.g., log-normal [2] and powerlaw [3]) and these inhomogeneous structures have remarkable effects on the dynamical processes on the substrate of networks [9, 10], especially for the dynamics of epidemics [1114]. Scores of researchers have proven that the strong heterogeneity of degree distribution can reduce or even vanish the epidemic threshold under some certain conditions [e.g., on the scale-free networks of degree distribution $p(k) \sim k^{-\gamma_{D}}$ with degree exponent $\gamma_{D} \leq 3$ in thermodynamic limit] [15, 16]. On weighted networks, some researches have shown that the inhomogeneity of weight distribution can also significantly affect the epidemic dynamics, such as the epidemic threshold and epidemic prevalence [17-24]. For instance, Zhou et al. suggested that increasing the dispersion of weight distribution can reduce the velocity of epidemic spreading as well as the epidemic prevalence [17, 18].

Moreover, these heterogeneous structural properties have

*Electronic address: tangminghuang521@ @otmail.com triggered the improvement of immunization strategy for complex networks. A few effective strategies have been proposed for the networks of heterogeneous degree distributions, including the targeted immunization strategy [25, 26], acquaintance immunization strategy [27] and information based immunization method [28]. For weighted networks, Deijfen proposed a variation of the so called acquaintance immunization strategy, where nodes are chosen randomly and these random nodes' neighbors with high edge-weights are vaccinated, and the modified strategy is more effective than the classical acquaintance immunization strategy where the neighbors are vaccinated randomly for a given vaccination coverage [21]. In addition, the targeted immunization strategy based on node's strength showed an effective immune effectiveness [29].

Most of the existing works studying epidemic dynamics and its immunization strategy on weighted networks have been analyzed through heterogeneous mean-field theory (HMF) [24, 30], percolation theory [21] or pairwise approximation method (PA) [22, 23]. The HMF theory assumes that the nodes of the same degrees will show the same dynamical characteristics [15, 31, 32], and can only qualitatively understand the effects of heterogeneous structural properties on quenched networks [24, 30]. Similar to the HMF theory, the analytical results derived from the percolation theory will also obviously deviate from the numerical results in the case of strong structural heterogeneity [21], which is caused by the strong dynamic correlations between two connected nodes [33]. The PA method can partly reflect the dynamic correlations and thus get a more accurate theoretical prediction [34, 35]. In the PA method, a number of $E \propto O\left(k_{\max }^{2} w_{\max }^{2}\right)$ equations is needed to govern the dynamical system, with $k_{\max }$ and $w_{\max }$ be the maximum degree and weight, respectively [22, 23]. So it will take a large amount of time to solve the nonlinear equations for epidemic dynamics when $k_{\max }$ and $w_{\max }$ are very large (i.e., networks with strong heterogenous degree and weight distributions), 
greatly limiting its ability of real-time prediction. Yang et al. developed an edge-based mean-field approximation to study epidemic spreading on homogeneous networks with heterogeneous weight distribution, but this method is not able to provide a very accurate prediction on reality weighted networks with strong structural heterogeneity [18]. Therefore, it is imperative for us to built a comprehensive method to depict the spreading dynamics on networks with general degree and weight distributions.

In this paper, we develop an edge-weight based compartmental approach to study epidemic spreading on networks with general degree and weight distributions. Our theory predicts that the epidemic threshold and epidemic size are closely related to the degree and weight distributions, which are in good agreement with the results from numerical simulations. In general, increasing the heterogeneity of weight distribution can suppress the epidemic spreading. However, for the degree distribution, increasing its heterogeneity can enhance (reduce) the epidemic size at the small (large) value of unit infection probability. We then propose an edge-weight based removal strategy to restrain the spreading of epidemic on weighted networks. Both the theoretical predications and experimental simulations indicate that an epidemic can be well controlled if the edges with high weights are preferentially removed, especially for the networks with the strong heterogenous weight distribution and homogenous degree distribution near the epidemic threshold.

The paper is organized as follows. In Sec. II, we describe weighted networks with general degree and weight distributions and the dynamical processes on it. In Sec. III, we will present an edge-weight based compartmental approach for the epidemic spreading and edge-weight based removal strategy on weighted networks. Numerical confirmation of the theoretical predictions will be provided in Sec. IV. We will draw our conclusions in Sec. V.

\section{MODEL}

We consider a population of size $N$ with degree distribution $p(k)$ and weight distribution $g(w)$. For the sake of simplicity, we assume that there is no correlation between the degree and weight distributions (i.e., edge weight is independent of node's degree). To construct a weighted network with the above degree and weight distributions, we first built an unweighted complex network as follows: i) generate a degree sequence following the degree distribution $p(k)$; ii) assign a total number of $k_{i}$ edge stubs to each node $i$; iii) randomly select two stubs to create an edge; iv) repeat the process iii) until there are no stubs left. Self-loops and multiple edges between the same pair of vertices are prohibited [36]. After that, each edge in the unweighted network is assigned a weight according to the weight distribution $g(w)$. The networks generated according to the above steps have no degree-degree and degree-weight correlations in the thermodynamic limit.

The epidemic spreading on weighted networks is described as a weighted Susceptible-Infected-Recovery (SIR) epidemiological model. In the SIR model, each node can be in one of the three states: susceptible state (S), infected state (I), and recovery state (R). To initiate an epidemic spreading process, a small number of nodes are randomly chosen to be infected and the other nodes are in susceptible state. At each time step, the disease first propagates from every infected node to all its neighbors. When a neighbor of one infected node is in the susceptible state, it will be infected with probability $\lambda(w)=1-(1-\beta)^{w}$, where $w$ is the weight of edge linking the two nodes and $\beta$ is the unit infection probability for $w=1$. Obviously, $\lambda(w)$ increases with $w$. If a susceptible node $i$ has $\Gamma_{i}$ infected neighbors, it will be infected with probability $1-\Pi_{j \in \Gamma_{i}}\left[1-\lambda\left(w_{i j}\right)\right]$, where $w_{i j}$ is the edge-weight between node $i$ and its infected neighbor $j$. At the same time step, each infected node can enter into the recovery state with probability $\gamma$. To be concrete, we set $\gamma=1.0$. Once an infected node is recovered, it will remain in this state for all subsequent times.

\section{EDGE-WEIGHT BASED COMPARTMENTAL APPROACH}

Two key quantities in the spreading dynamics are the epidemic threshold and epidemic size (i.e., final infected density). We first develop an edge-weight based compartmental approach to predict these two quantities on the networks with arbitrary degree and weight distributions. Then, we investigate the effectiveness of the edge-weight based removal strategy on epidemic spreading through the proposed method. The time evolution of the epidemic spreading is described by the variables $S(t), I(t)$ and $R(t)$, which are the densities of the susceptible, infected, and recovered nodes at time $t$, respectively.

\section{A. Spreading dynamics}

In the classical heterogeneous mean-field theory (CHMF), nodes are classified according to their degrees, which means all the nodes within a given class are considered to be statistically equivalent [31, 32]. However, apart from the heterogeneity of degree distribution, the heterogeneity of weights on edges makes the CHMF theory be hard to accurately describe the spreading dynamics on weighted networks [30]. To solve this question, we develop an edge-weight based compartmental theory, which is inspired by Refs. [37, 38].

We define $\theta_{w}(t)$ to be the probability that a node $v$ has not transmitted the infection to a node $u$ along a randomly chosen edge with weight $w$. Initially, only a few nodes are in the infected state, which means $\theta_{w}(t)$ is close to unity. A randomly selected node $u$ is not infected by one of its neighbors with probability

$$
\theta(t)=\sum_{w} g(w) \theta_{w}(t)
$$

By time $t$, if none of its neighbor has transmitted the infection to node $u$, it will remain in the susceptible state. Supposing its degree is $k$, it is susceptible at time $t$ with probability $\theta(t)^{k}$. 
Thus, the proportion of the susceptible nodes (i.e., the probability that a randomly selected node is susceptible) at time $t$ is

$$
S(t)=\sum_{k=0} p(k) \theta(t)^{k}=G(\theta(t)),
$$

where $G(x)=\sum_{k} p(k) x^{k}$ is the generating function for degree distribution.

A neighbor of node $u$ may be in one of susceptible, infected and recovered states, and thus the probability $\theta_{w}(t)$ for weight $w$ can be divided into three parts:

$$
\theta_{w}(t)=\xi_{w}^{S}(t)+\xi_{w}^{I}(t)+\xi_{w}^{R}(t),
$$

where $\xi_{w}^{S}(t)\left(\xi_{w}^{I}(t)\right.$ or $\left.\xi_{w}^{R}(t)\right)$ is denoted as the probability that a neighbor is in the susceptible (infected or recovery) state and has not transmitted the infection to node $u$ through an edge with weight $w$ by time $t$. Once these three parameters can be derived, we will get the density of susceptible nodes at time $t$ by substituting them into Eq. (1) and then into Eq. (2). To this purpose, in the following, we will focus on how to solve them.

If a neighbor of node $u$ is susceptible, it can not infect the node $u$, and vice versa. On the uncorrelated networks, one link of node $u$ connects to a node with degree $k$ with probability $k p(k) /\langle k\rangle$, where $\langle k\rangle$ is the mean degree of a network [39]. In the mean-field level, the probability that one of its neighbors is in susceptible state is $\xi_{w}^{S}(t)=\Sigma_{k} k p(k) \theta(t)^{k-1} /\langle k\rangle$. Utilizing the generating function for degree distribution $G(x)$, we have

$$
\xi_{w}^{S}(t)=\frac{G^{\prime}(\theta(t))}{G^{\prime}(1)} .
$$

According to the spreading process described in Sec. II we know that the growth of $\xi_{w}^{R}(t)$ includes two consecutive events: firstly, an infected neighbor has not transmitted the infection to node $u$ via their edge with weight $w$, with probability $1-\lambda(w)$; secondly, the infected neighbor has been recovered, with probability $\gamma=1.0$. Combining these two events, we have

$$
\frac{d \xi_{w}^{R}(t)}{d t}=(1-\lambda(w)) \xi_{w}^{I}(t) .
$$

If this infected neighbor transmits the infection via an edge with weight $w$, the rate of flow from $\theta_{w}(t)$ to $1-\theta_{w}(t)$ will be $\lambda(w) \xi_{w}^{I}(t)$, which means

$$
\frac{d \theta_{w}(t)}{d t}=-\lambda(w) \xi_{w}^{I}(t)
$$

and

$$
\frac{d\left(1-\theta_{w}(t)\right)}{d t}=\lambda(w) \xi_{w}^{I}(t) .
$$

By combining Eqs. (5) and (7), one obtains

$$
\xi_{w}^{R}=\frac{\left(1-\theta_{w}(t)\right)(1-\lambda(w))}{\lambda(w)} .
$$

Substituting Eq. (4) of $\xi_{w}^{S}(t)$ and Eq. (8) of $\xi_{w}^{R}(t)$ into Eq. (3), we yield the following relation

$$
\xi_{w}^{I}(t)=\theta_{w}(t)-\frac{G^{\prime}(\theta(t))}{G^{\prime}(1)}-\left(1-\theta_{w}(t)\right) \frac{1-\lambda(w)}{\lambda(w)} .
$$

Injecting Eq. (9) into Eq. (6), we have

$$
\frac{d \theta_{w}(t)}{d t}=\lambda(w) \frac{G^{\prime}(\theta(t))}{G^{\prime}(1)}+1-\lambda(w)-\theta_{w}(t) .
$$

From Eq. (10), the probability $\theta_{w}(t)$ can be solved. Substituting the value of $\theta_{w}(t)$ into Eqs. (1) and (2), the density associated with each distinct state is given by

$$
\begin{aligned}
\frac{d R(t)}{d t} & =I(t), \\
S(t) & =G(\theta(t)), \\
I(t) & =1-R(t)-S(t) .
\end{aligned}
$$

According to Eqs. (10)-13), one can find that only $E \propto$ $O\left(w_{\max }\right)$ equations are required in the edge-weight based compartmental approach to describe the dynamics of epidemic on networks with arbitrary degree and weight distributions. By setting $t \rightarrow \infty$ and $d \theta_{w} / d t=0$ in Eq. (10), we get the probability of one edge with weight $w$ which does not propagate disease in the spreading process

$$
\theta_{w}(\infty)=\lambda(w) \frac{G^{\prime}(\theta(\infty))}{G^{\prime}(1)}+1-\lambda(w) .
$$

Substituting $\theta_{w}(\infty)$ into Eqs. (1) and (2), we can figure out the value of $S(\infty)$, and then the epidemic size $R(\infty)$ can be obtained.

Another important issue in epidemic spreading is to determine the epidemic threshold. Below the epidemic threshold, the epidemic will die out; otherwise, the epidemic will spread and become possible. To this end, we summate $\theta_{w}(\infty)$ for all possible $w$ in Eq. (14) and obtain

$$
\theta(\infty)=\langle\lambda(w)\rangle \frac{G^{\prime}(\theta(\infty))}{G^{\prime}(1)}+1-\langle\lambda(w)\rangle,
$$

where

$$
\langle\lambda(w)\rangle=\sum_{w} g(w) \lambda(w)
$$

is the mean transmission rate for a randomly selected edge. Indeed, we can obtain the threshold of epidemic by observing where the non-trivial solution of Eq. (15) appears, corresponding to the point at which the right-hand side of the equation is tangent to the line $y=\theta(\infty)$ at $\theta(\infty)=1$ [36]. The condition of the epidemic threshold is thus given by

$$
\left\langle\lambda_{c}(w)\right\rangle=\frac{G^{\prime}(1)}{G^{\prime \prime}(1)}=\frac{\langle k\rangle}{\left\langle k^{2}\right\rangle-\langle k\rangle} .
$$

Further solving the above equation, one can get the epidemic threshold

$$
\beta_{c}=1-F^{-1}(z)
$$


where $F(x)=\sum_{w} g(w) x^{w}$ is the generating function for weight distribution, $F^{-1}(z)$ is the inverse function of $F(x)$, and $z=1-\langle k\rangle /\left(\left\langle k^{2}\right\rangle-\langle k\rangle\right)$.

From Eq. [18), we see that the epidemic threshold is closely related to the degree and weight distributions. For a given weight distribution, the stronger heterogeneity of degree distribution with the larger value of $z$ results in the smaller value of $\beta_{c}$, as $F^{-1}(z)$ is a monotone increasing function. By contrast, increasing the heterogeneity of weight distribution can enhance the threshold of epidemic outbreak when the degree distribution is fixed, since $F^{-1}(z)$ decreases with the heterogeneity of weight distribution at $0<z<1$. If the weight on every edge equals to unity, the epidemic threshold will return to the result obtained on unweighted networks [40].

\section{B. Effectiveness of edge removal}

To prevent an epidemic in time, different strategies to immunize nodes or edges of a network have been widely studied [27, 41]. A successful immunization strategy must be able to accurately identify and immunize the influential nodes or edges in the process of epidemic spreading, which can significantly enhance the epidemic threshold and reduce the epidemic size [42]. In weighted networks, the edge weight reflects the relative importance of the connections between nodes, and edges with high weights may play a more significant role in the spreading process [43]. In the ideal case with full knowledge of all edge weights, removing the edges with high weights can prevent epidemic spreading more efficiently. But in many realistic cases, this complete information is not available, and only partial knowledge exists [44-46]. Therefore, we here focus on a general edge-weight based removal model with the family of function [46, 47]

$$
\Phi(w)=\frac{w^{\alpha}}{\sum_{i=1}^{M} w_{i}^{\alpha}},-\infty<\alpha<+\infty,
$$

where a value $\Phi(w)$ is assigned to each edge, which stands for the probability that an edge with weight $w$ is removed, $M$ is the total number of edges, and $\alpha$ is an exponent of preferential removal. For $\alpha=0.0$, we have $\Phi(w)=1 / M$, which means every edge has the same removal probability. The case of $\alpha \rightarrow+\infty$ represents that the strategy is to remove edges in a descending order (i.e., from edges with high weights to edges with low weights). For $\alpha \rightarrow-\infty$, the opposite case happens. After an edge removal strategy is executed, where a fraction $1-f$ of edges are removed from the original network according to Eq. [19, we initiate an infection on the residual network.

To obtain the epidemic threshold and epidemic size, we first figure out the degree and weight distributions of the residual network, and then solve these two key quantities through the edge-weight based compartmental theory in Sec. III. A. In our network model, degree and weight distributions are respectively independent, which means a fraction of $1-f$ edges are randomly removed in the edge removal strategies with different values of $\alpha$. Equivalently, the residual network can be gotten by randomly occupying a fraction of $f$ edges in the original network. Using the percolation theory, the degree distribution of the residual network is given by [36, 40]

$$
p_{f}(k)=\sum_{m=k} p(m)\left(\begin{array}{c}
m \\
k
\end{array}\right) f^{k}(1-f)^{m-k} .
$$

Letting $A_{f}(w)$ be the number of edges with weight $w$ and $g_{f}(w)$ be the residual weight distribution in the residual weighted network with the remaining fraction $f$ of edges, we have the residual weight distribution as

$$
g_{f}(w)=\frac{A_{f}(w)}{f M} .
$$

When one additional edge is removed by implementing the edge weight based removal strategy as Eq. [19, $A_{f}(w)$ becomes

$$
A_{\left(f-\frac{1}{M}\right)}(w)=A_{f}(w)-\frac{g_{f}(w) w^{\alpha}}{\left\langle w^{\alpha}(f)\right\rangle},
$$

where $\left\langle w^{\alpha}(f)\right\rangle=\sum_{w} g_{f}(w) w^{\alpha}$. In the thermodynamic limit $M \rightarrow \infty$, Eq. (22) can be presented in terms of derivative of $A_{f}(k)$ with respect to $f$,

$$
\frac{d A_{f}(w)}{d f}=M \frac{g_{f}(w) w^{\alpha}}{\left\langle w^{\alpha}(f)\right\rangle} .
$$

Differentiating Eq. 21) with respect to $f$ and substituting it into Eq. (23), we obtain

$$
-f \frac{d g_{f}(w)}{d f}=g_{f}(w)-\frac{g_{f}(w) w^{\alpha}}{\left\langle w^{\alpha}(f)\right\rangle} .
$$

To solve Eq. 24, we define a function $H_{\alpha}(t)=$ $\sum_{w} g(w) t^{w^{\alpha}}$, and let $t=H_{\alpha}^{-1}(f)$. We find by direct differentiation that [47, 48]

$$
g_{f}(w)=g(w) \frac{t^{w^{\alpha}}}{H_{\alpha}(t)}=\frac{1}{f} g(w) t^{w^{\alpha}},
$$

and

$$
\left\langle w^{\alpha}(f)\right\rangle=\frac{t H_{\alpha}^{\prime}(t)}{H_{\alpha}(t)} .
$$

From Eqs. (20) and (25), we can get the degree and weight distributions of the residual weighted network, respectively. Substituting them into Eqs. (11)-(14) and Eq. (18), we can obtain the epidemic size and outbreak condition on the residual network, respectively.

\section{NUMERICAL SIMULATIONS}

In simulations, the size of networks, the mean degree and the mean edge weight are set to be $N=10^{4},\langle k\rangle=10$ and $\langle w\rangle=8$, respectively. Without of lose generality, we consider the networks with degree distribution $p(k) \sim k^{-\gamma_{D}}$ and weight distribution $g(w) \sim w^{-\gamma_{W}}$ to verify the theoretical 
approach, where $\gamma_{D}$ and $\gamma_{W}$ represent degree and weight exponents, respectively. The smaller values of the exponents, the more heterogeneous of the distributions [49]. The maximum degree and weight are set to be $k_{\max } \sim \sqrt{N}$ [50] and $w_{\max } \sim N^{1 /\left(\gamma_{W}-1\right)}$ [18], respectively. To initiate an infection process, we randomly choose five infected nodes as seeds, while the other nodes are in the susceptible state.

We employ the susceptibility measure [33, 51] $\chi$ to numerically determine the epidemic threshold

$$
\chi=N \frac{\left\langle r^{2}\right\rangle-\langle r\rangle^{2}}{\langle r\rangle},
$$

where $r$ denotes the epidemic size $R$. To obtain a reliable value of $\chi$, we use at least $2 \times 10^{3}$ independent dynamic realizations on a fixed weighted network to calculate the average value of $\chi$ for each value of unit infection probability $\beta$. Susceptibility $\chi$ exhibits a maximum value at $\beta_{c}$, which is the threshold value of the epidemic spreading process. The simulations are further implemented by using 100 different network realizations to obtain the mean threshold $\beta_{c}$. The identical simulation setting is used for all subsequent numerical results, unless otherwise specified.

We first investigate the influence of degree distribution on the epidemic dynamics. Fig. 1 (a) shows that the epidemic threshold $\beta_{c}$ decreases with the heterogeneity of degree distribution (i.e., the smaller value of $\gamma_{D}$ ) on the weighted networks, which is consistent with the epidemic outbreak on the unweighted networks [15]. This reason stems from the existence of more hub nodes on strong heterogeneous networks. However, the effect of degree distribution on the epidemic size $R(\infty)$ is more complex. As shown in Fig. 1 (b), increasing the heterogeneity of degree distribution can promote the epidemic size at small $\beta$ while suppress the epidemic size at large $\beta$. For instance, at a fixed value of $\gamma_{W}=2.1$, the epidemic size $R(\infty)$ for $\gamma_{D}=2.1$ is greater than that for $\gamma_{D}=4.0$ when $\beta \leq 0.03$ (i.e., promotion region), while the situation is exactly opposite when $\beta>0.03$ (i.e., suppression region). This result can be qualitatively explained as follows: epidemic propagates on complex networks following a hierarchical way. That is to say, the hubs with large degrees are more likely to become infected at the early times of epidemic spreading [52]. For a small value of $\beta$, the existence of hubs makes the epidemic spread more easily. But for a large value of $\beta$, more nodes with small degrees in more heterogeneous networks have a small infection probability, which results in the lower $R(\infty)$.

The effects of heterogeneity of weight distribution on the epidemic threshold as well as the epidemic size are also given in Fig. 11 One can find that, when the degree distribution (i.e., the value of $\gamma_{D}$ ) is fixed, increasing the heterogeneity of weight distribution (i.e., decreasing the value of $\gamma_{W}$ ) not only enhances the epidemic threshold $\beta_{c}$ [see Fig. 11a)] but also reduces the epidemic size $R(\infty)$ [see Fig. 1 b)]. This phenomenon can be explained as follows: when the average weight $\langle w\rangle$ is fixed, the small value of $\gamma_{W}$ causes most edges possessing lower weights and infection probabilities, leading to the fact that the mean transmission rate $\langle\lambda(w)\rangle$ for a randomly selected edge is smaller on the networks with more het-
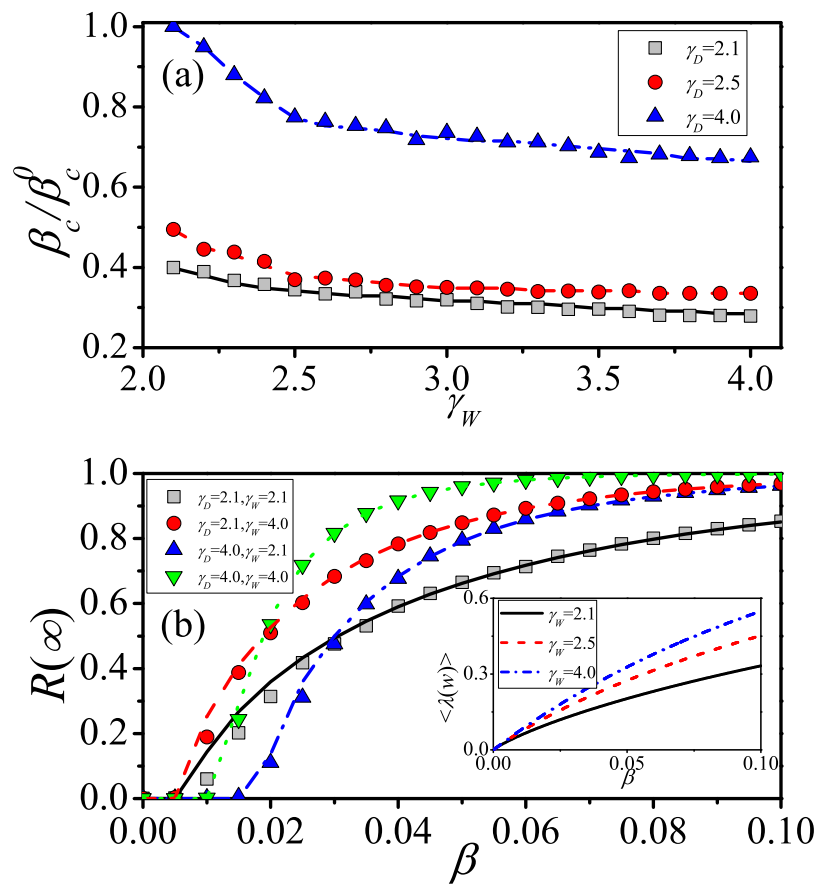

FIG. 1: (Color online) The influence of degree and weight distributions on the epidemic threshold and epidemic size. (a) The relative epidemic threshold $\beta_{c} / \beta_{c}^{0}$ as a function of weight exponent $\gamma_{W}$ for degree exponents $\gamma_{D}=2.1$ (gray squares), $\gamma_{D}=2.5$ (red circles) and $\gamma_{D}=4.0$ (blue up triangles), where $\beta_{c}^{0} \approx 0.016$ is the theoretical threshold at $\gamma_{D}=4.0, \gamma_{W}=2.1$. Black solid, red dashed and blue dot-dashed lines are the numerical solutions from Eq. (18). (b) Epidemic size $R(\infty)$ versus unit infection probability $\beta$ for $\gamma_{D}=2.1, \gamma_{W}=2.1$ (gray squares), $\gamma_{D}=2.1, \gamma_{W}=$ 4.0 (red circles), $\gamma_{D}=4.0, \gamma_{W}=2.1$ (blue up triangles) and $\gamma_{D}=4.0, \gamma_{W}=4.0$ (green down triangles). Black solid, red dashed, blue dot-dashed and green dot lines are the numerical solutions from Eqs. (11)-(14). The inset of (b) shows the numerical solutions of $\langle\lambda(w)\rangle$ from Eq. 16 as function of $\beta$ for three different values of $\gamma_{W}$ (i.e., 2.1, 2.5, and 4.0), corresponding to the black solid, red dashed and blue dot-dashed lines.

erogeneous weight distribution [see Eq. (16) and its numerical solutions in the inset of Fig. 11 (b)]. In addition, from Fig. 11a) one can see that the epidemic threshold $\beta_{c}$ increases more remarkably when $\gamma_{W} \leq 2.5$ due to the strong heterogeneity of the weight distribution. On these networks with strong heterogeneous degree and weight distributions, the developed edgeweight based approach can still accurately reproduce the simulated $\beta_{c}$ and $R(\infty)$ in Fig. 1.

To further investigate the impacts of the two heterogeneous distributions on the epidemic size, $R(\infty)$ as a function of the exponents $\gamma_{D}$ and $\gamma_{W}$ is shown in Fig. 2] where the unit infection probability is set to $\beta=0.04$ in the suppression region for ensuring the outbreak of epidemic. From Figs. 2 (a) and (b), we see that the weaker heterogeneity of degree and weight distributions can lead to the higher epidemic size, that is, $R(\infty)$ increases with the growth of $\gamma_{D}$ and $\gamma_{W}$. We also show the 

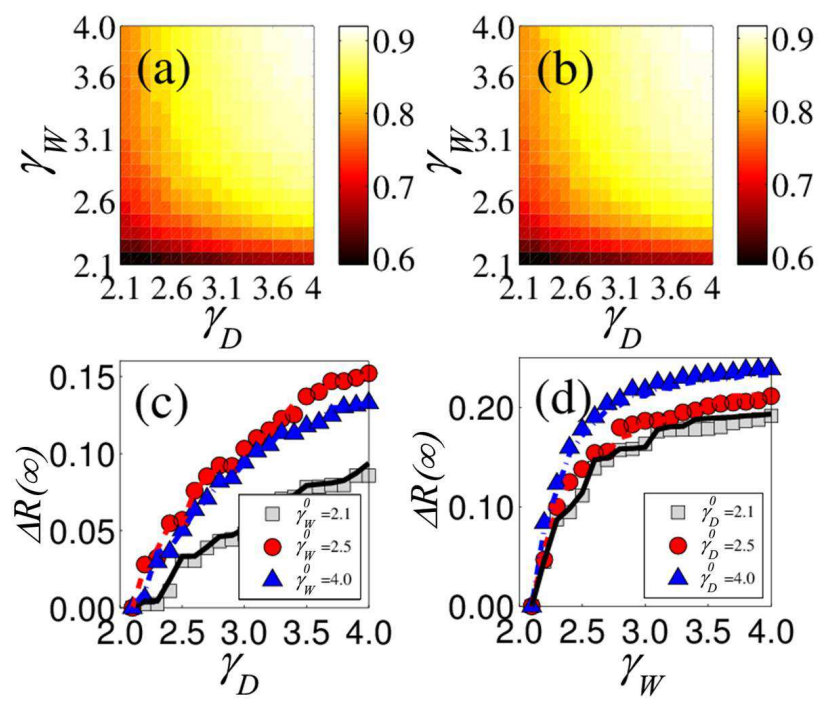

FIG. 2: (Color online) Epidemic size changes with the degree and weight exponents. Color-coded values of epidemic size from numerical simulations (a) and theoretical solutions (b) are shown on the $\gamma_{D}, \gamma_{W}$ plane. (c) The increment of epidemic size $\Delta R\left(\gamma_{D}, \gamma_{W}^{0}, \infty\right)$ as a function of degree exponent $\gamma_{D}$ at weight exponents $\gamma_{W}^{0}=$ 2.1 (gray squares), $\gamma_{W}^{0}=2.5$ (red circles) and $\gamma_{W}^{0}=4.0$ (blue up triangles). (d) $\Delta R\left(\gamma_{D}^{0}, \gamma_{W}, \infty\right)$ as a function of $\gamma_{W}$ at $\gamma_{D}^{0}=$ 2.1 (gray squares), $\gamma_{D}^{0}=2.5$ (red circles) and $\gamma_{D}^{0}=4.0$ (blue up triangles). The values in (b) and the lines in (c) and (d) are the numerical solutions of Eqs. (11)-14 in the limit $t \rightarrow \infty$. The unit infection probability is set to $\beta=0.04$.

increase of $R(\infty)$ with $\gamma_{D}\left(\gamma_{W}\right)$ at a fixed value of $\gamma_{W}^{0}\left(\gamma_{D}^{0}\right)$ in Fig. 2 (c) [Fig. 22(d)]. Defining the increment of $R(\infty)$ as $\Delta R\left(\gamma_{D}, \gamma_{W}, \infty\right)=R\left(\gamma_{D}, \gamma_{W}, \infty\right)-R\left(\gamma_{D}^{0}, \gamma_{W}^{0}, \infty\right)$, where $R\left(\gamma_{D}, \gamma_{W}, \infty\right)$ is the epidemic size for a $\left(\gamma_{D}, \gamma_{W}\right)$ pair, we can investigate the impact of one parameter on the value of $\Delta R\left(\gamma_{D}, \gamma_{W}, \infty\right)$ by fixing the other parameter. For example, by setting $\gamma_{W}^{0}=2.1$ we can look into how $\Delta R\left(\gamma_{D}, \gamma_{W}^{0}, \infty\right)$ changes with $\gamma_{D}$. We note that the increment of $R(\infty)$ tends to be more evident for the more homogeneous weight and degree distributions (i.e., greater $\gamma_{W}$ and $\gamma_{D}$ ), which results from the greater mean transmission rate $\langle\lambda(w)\rangle$ and fewer nodes with small degrees having a small infection probability, respectively. From Fig. 2 we see that the theoretical predictions are in good agreement with the simulated epidemic size, no matter how heterogeneous the degree and weight distributions are.

In the following, we check the effectiveness of the edgeweight based removal strategy on controlling epidemics. Fig. 3 reports the epidemic threshold and epidemic size as a function of the tunable parameter $\alpha$ when a fraction $1-f=$ 0.2 of edges are removed according to Eq. 19). For $\alpha>0.0$, preferentially removing edges with high weights can partly restrain the spread of epidemic (i.e., enhance the epidemic threshold and reduce the epidemic size). The reason of this phenomenon is that the removal of strong ties (i.e., edges with high weights) can reduce the value of $\langle\lambda(w)\rangle$ more effectively than that of weak ties (i.e., edges with low weights). So the
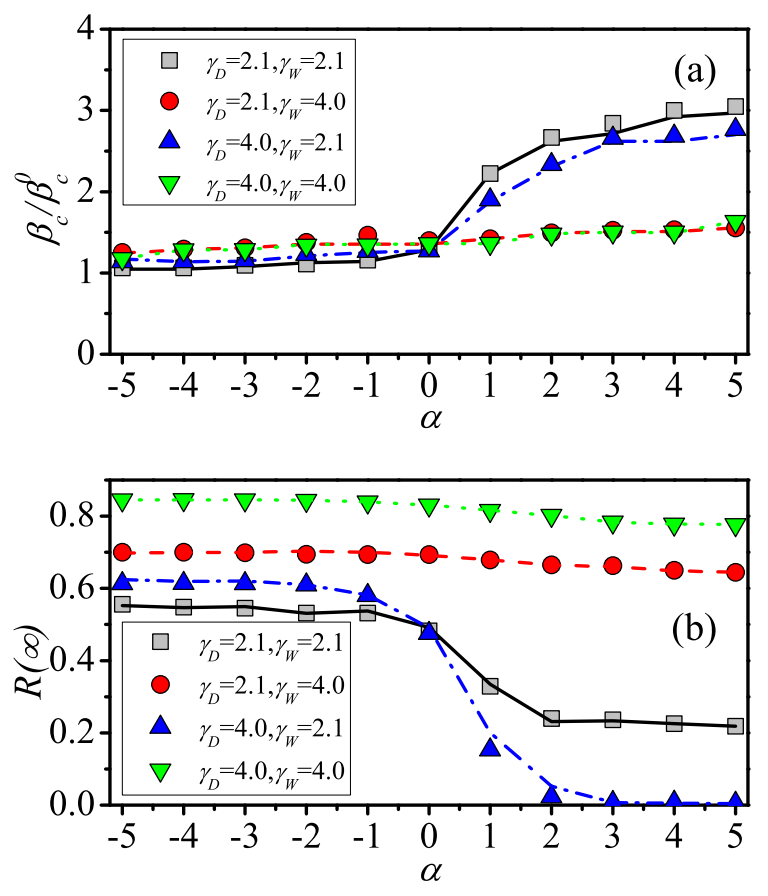

FIG. 3: (Color online) The control effectiveness of the edge-weight based removal strategy with different bias. The relative epidemic threshold $\beta_{c} / \beta_{c}^{0}$ (a) and epidemic size $R(\infty)$ (b) as a function of parameter $\alpha$ on different networks with tunable parameters $\gamma_{D}=$ $2.1, \gamma_{W}=2.1$ (gray squares), $\gamma_{D}=2.1, \gamma_{W}=4.0$ (red circles), $\gamma_{D}=4.0, \gamma_{W}=2.1$ (blue up triangles) and $\gamma_{D}=4.0, \gamma_{W}=$ 4.0 (green down triangles). Black solid, red dashed, blue dot-dashed and green dot lines are the analytical predictions from Eq. 18) for the relative threshold and Eqs. (11)-14 for the epidemic size, with the degree and weight distributions according to Eqs. 20) and 25, respectively. In subfigure (a), $\beta_{c}^{0}$ is the theoretical threshold on the original network.

control effect for $\alpha \leq 0.0$ is negligible, as the removal of edges is concentrated on the weak ties. In addition, one can see that the more heterogenous the weight distribution is (i.e., the smaller value of $\gamma_{W}$ ), the better effectiveness the edgeweight based removal strategy plays when $\alpha>0.0$. For $\gamma_{W}=2.1$ in Fig. 3 (a), the edge removal strategy with large $\alpha$ makes the epidemic threshold $\beta_{c}$ increases by two or three times, that is the relative threshold $\beta_{c} / \beta_{c}^{0} \approx 2.5$, where $\beta_{c}$ and $\beta_{c}^{0}$ are respectively the epidemic thresholds for the residual and original networks. Fig. 3 (b) also shows that the epidemic can almost be eliminated on the networks with $\gamma_{D}=4.0$ and $\gamma_{W}=2.1$ when $\alpha \geq 2.0$ [see the blue up triangles in Fig. 3 (b)], because the epidemic threshold $\beta_{c}$ is close to 0.04 after removing many strong ties [see Fig. 3(a)].

We further address the performance of this removal strategy on reducing the epidemic size for $\beta=0.04$ in Fig. 4. The decrement of epidemic size is defined as $\Delta R^{\prime}\left(\gamma_{D}, \gamma_{W}, \infty\right)=$ $R_{0}\left(\gamma_{D}, \gamma_{W}, \infty\right)-R\left(\gamma_{D}, \gamma_{W}, \infty\right)$, where $R_{0}\left(\gamma_{D}, \gamma_{W}, \infty\right)$ and $R\left(\gamma_{D}, \gamma_{W}, \infty\right)$ are the epidemic sizes on the original network and residual network, respectively. Figs. 4 (a) and (b) 


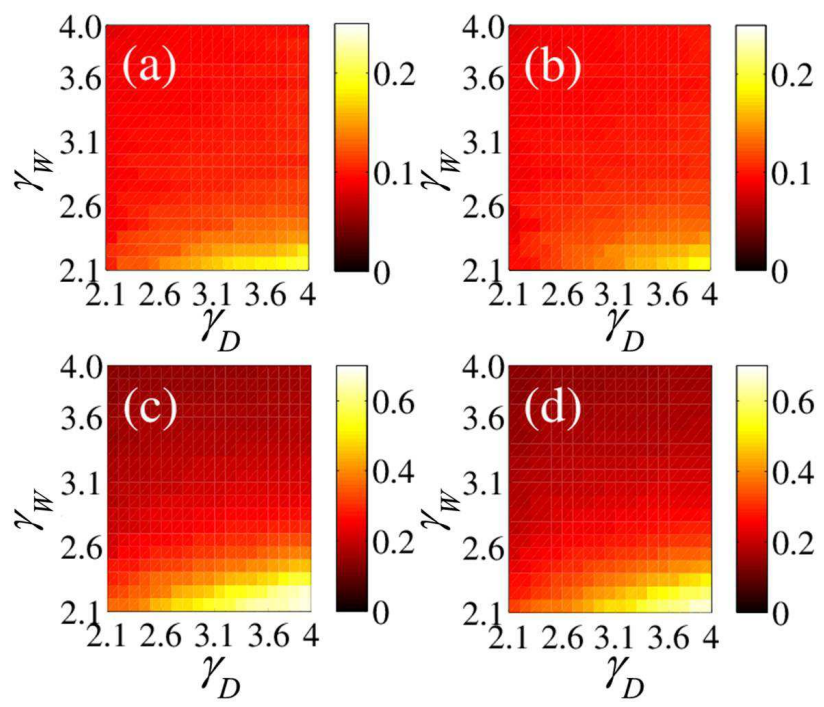

FIG. 4: (Color online) The decrement of epidemic size $\triangle R^{\prime}(\infty)$ as a function of $\gamma_{D}$ and $\gamma_{W}$ for different values of $\alpha$. (a) and (c) represent respectively the numerical simulations for $\alpha=0.0$ and $\alpha=5.0$, and the theoretical predications for $\alpha=0.0$ and $\alpha=5.0$ are shown in (b) and (d), respectively. The unit infection probability is $\beta=0.04$.

reveal that, $\Delta R^{\prime}\left(\gamma_{D}, \gamma_{W}, \infty\right)$ is small for $\alpha=0.0$, as the value of $\langle\lambda(w)\rangle$ is little changed and the mean degree of the residual network $\langle k\rangle$ is slightly smaller, when some edges are randomly removed. We also see that the $\Delta R^{\prime}\left(\gamma_{D}, \gamma_{W}, \infty\right)$ is smaller for the case of small $\gamma_{D}$ when $\gamma_{W}$ is small, as the existence of hubs makes the epidemic spreading near the threshold has a better robustness against random edge failures [36]. In Figs. 4 (c) and (d), the edge-weight based removal strategy with $\alpha=5.0$ is more effective, especially for the networks with strong heterogeneous weight distribution, which results from the $\langle\lambda(w)\rangle$ decreasing faster.

Moreover, we study the influence of edge removal proportion on the epidemic threshold for $\alpha=0.0$ [see Fig. [5 (a)] and $\alpha=5.0$ [see Fig. 5](b)]. For comparison, we define the relative threshold $\beta_{c} / \beta_{c}^{0}$ as the ratio of the threshold $\beta_{c}$ on the residual network to the threshold $\beta_{c}^{0}$ on the original network. As is expected, increasing the immunization proportion $1-f$ results in the increase of the relative threshold $\beta_{c} / \beta_{c}^{0}$. We also note that the targeted edge removal strategy with $\alpha=5.0$ presents a much better performance on the weighted networks with small $\gamma_{W}$, e.g., $\beta_{c} / \beta_{c}^{0}\left(\gamma_{W}=4.0\right)<\beta_{c} / \beta_{c}^{0}\left(\gamma_{W}=2.1\right)$ in Fig.5(b). What's more, the simulated results are well fitted by the theoretical predictions presented in Sec. IIIB

\section{CONCLUSIONS}

In sum, in this paper, we developed an edge-weight based approach to describe the spread of epidemic on the networks with heterogenous degree distribution as well as heterogenous weight distribution. Our findings indicate that the predictions from such a method can be in good agreement with the sim-
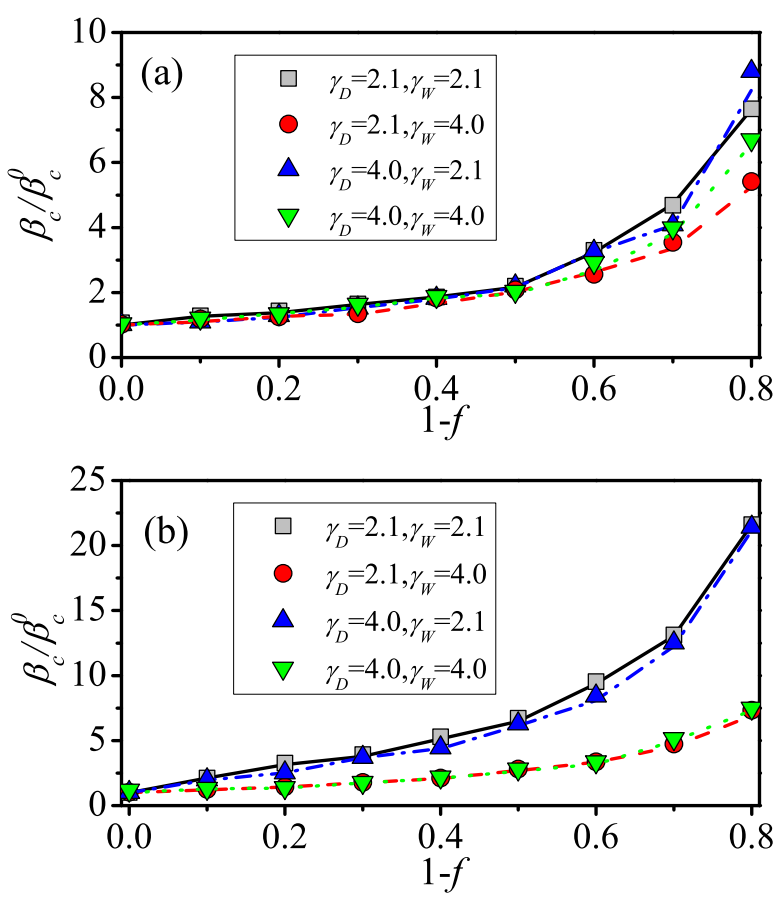

FIG. 5: (Color online) The relative epidemic threshold as a function of removal proportion $1-f$ for (a) $\alpha=0.0$ and (b) $\alpha=5.0$ on different networks, including the parameters $\gamma_{D}=2.1, \gamma_{W}=$ 2.1 (gray squares), $\gamma_{D}=2.1, \gamma_{W}=4.0$ (red circles), $\gamma_{D}=$ $4.0, \gamma_{W}=2.1$ (blue up triangles) and $\gamma_{D}=4.0, \gamma_{W}=4.0$ (green down triangles). Black solid, red dashed, blue dot-dashed and green dot lines are the analytical predictions from Eq. 18 with the degree and weight distributions according to Eqs. 20) and 25, respectively. And the parameter $\beta_{c}^{0}$ is the theoretical threshold on the original network.

ulated epidemic threshold and epidemic size. Combing the numerical simulations and the theoretical analysis, we found that the strong heterogeneity of degree distribution and the weak heterogeneity of weight distribution can both make networks be more fragile to the outbreak of epidemics. Unlike the effect of weak heterogeneity of weight distribution which always promotes epidemic spreading, the effects of the heterogeneity of degree distribution on the epidemic size can be divided into two distinct regions: the strong heterogeneity of degree distribution promotes the epidemic size when the unit infection probability is small, on the contrary, the strong heterogeneity suppresses the epidemic size at a large unit infection probability. Thus, for a large value of unit infection probability the epidemic spreading will be mostly promoted once both the degree distribution and the weight distribution are more homogenous. Moreover, we proposed an edge-weight based removal strategy and investigated the effectiveness of this strategy on epidemic control. Generally speaking, removing edges with high weights is more effective to suppress epidemic spreading on the networks with strong heterogeneous weight distribution, especially for the networks having more homogeneous degree distribution near the epidemic threshold. 
We here provides a more accurate theoretical framework to solve the epidemic spreading on complex networks with general degree and weight distributions, which could be applied to other analogous dynamical processes such as information diffusion and cascading failure. Besides, how to develop an analytic method for being suitable for the case of the correlation between nodes' degrees and edge weights existing still needs to think deeply. This work helps to understand the spreading dynamics on heterogeneous weighted networks in depth and would stimulate further works in designing better immunization strategies.

\section{Acknowledgments}

This work was partially supported by National Natural Science Foundation of China (Grant Nos. 11105025, 11135001,
91324002, 11331009), China Postdoctoral Science Special Foundation (Grant No. 2012T50711), the Program of Outstanding Ph. D. Candidate in Academic Research by UESTC (Grand No. YXBSZC20131065). Y. Do was supported by Basic Science Research Program through the National Research Foundation of Korea (NRF) funded by the Ministry of Education, Science and Technology (NRF-2013R1A1A2010067).
[1] http://en.wikipedia.org/wiki/Weighted_network

[2] M. E. J. Newman, Proc. Natl. Acad. Sci. USA 98, 404 (2001).

[3] A. Barrat, M. Barthélemy, R. Pastor-Satorras, and A. Vespignani, Proc. Natl. Acad. Sci. USA 101, 3747 (2004).

[4] J. P. Onnela, J. Saramki, J. Hyvnen, G. Szab, M. A. Menezes, K. Kaski, A. L. Barabsi, and J. Kertsz, New J. Phys. 9, 179 (2007).

[5] Z. Zhuo, S.-M. Cai, Z.-Q. Fu, and J. Zhang, Phys. Rev. E 84, 031923 (2011).

[6] L. A. Amaral, A. Scala, M. Barthélemy, and H. E. Stanley, Proc. Natl Acad. Sci. USA 97, 11149 (2000).

[7] L. E. C. daRocha, J. Stat. Mech. P04020 (2009).

[8] R. Albert and A.-L. Barabási, Rev. Mod. Phys. 74, 47 (2002).

[9] S. N. Dorogovtsev, A. V. Goltsev, and J. F. F. Mendes, Rev. Mod. Phys. 80, 1275 (2008).

[10] P. Holme, B. J. Kim, C. N. Yoon, and S. K. Han, Phys. Rev. E 65, 056109 (2002).

[11] M. E. J. Newman, SIAM Rev. 45, 167 (2003).

[12] L. Wang and X. Li, Chin. Sci. Bull. 10, 1007 (2014).

[13] T. Gross and B. Blasius, J. R. Soc. Interface 5, 259 (2008).

[14] P. Holme and J. Saramaki, Phys. Rep. 519, 97 (2012).

[15] R. Pastor-Satorras and A. Vespignani, Phys. Rev. Lett. 86, 3200 (2001).

[16] M. Boguñá, C. Castellano, and R. Pastor-Satorras, Phys. Rev. Lett. 111, 068701 (2013).

[17] G. Yan, T. Zhou, J. Wang, Z. Q. Fu, and B. H. Wang, Chin. Phys. Lett. 22, 510 (2005).

[18] Z. Yang and T. Zhou, Phys. Rev. E 85, 056106 (2012).

[19] X. Chu, J. Guan, Z. Zhang, and S. Zhou, J. Stat. Mech. 07043 (2009).

[20] Y. Min, X. Jin, Y. Ge, and J. Chang, PLoS ONE, 8, e57100 (2013).

[21] M. Deijfen, Mathematical Biosciences. 232, 57 (2011).

[22] C. Kamp, M. Moslonka-Lefebvrey, and S. Alizon, PLoS Comput. Biol. 9, e1003352 (2013).

[23] P. Rattana, K. B. Blyuss, K. T. D. Eames, and I. Z. Kiss, Bull. Math. Biol. 75, 466 (2013).

[24] Y. Sun, C. Liu, C.-X. Zhang, and Z.-K. Zhang, Phys. Lett. A 378, 635C640 (2014).

[25] J. Gómez-Gardeñes, P. Echenique, and Y. Moreno, Eur. Phys. J. B 49, 259-264(2006).
[26] H. F. Zhang, K.-Z. Li, X.-C. Fu, and B.-H. Wang, Chin. Phys. Lett. 26, 068901 (2009).

[27] R. Cohen, S. Havlin, and D. ben-Avraham, Phys. Rev. Lett. 91, 247901 (2003).

[28] C. Granell, S. Gómez, and A. Arenas, Phys. Rev. Lett. 111, 128701 (2013).

[29] K. T. D. Eames, J. M. Read, and W. J. Edmunds. Epidemics 1, 70 (2009).

[30] C. Buono, F. Vazquez, P. A. Macri, and L. A. Braunstein, Phys. Rev. E 88, 022813 (2013).

[31] Y. Moreno, R. Pastor-Satorras, and A. Vespignani, Eur. Phys. J. B 26, 521 (2002).

[32] C. Castellano and R Pastor-Satorras, Phys. Rev. Lett. 96, 038701 (2006).

[33] S. C. Ferreira, C. Castellano, and R. Pastor-Satorras, Phys. Rev. E 86, 041125 (2012).

[34] K. T. D. Eames and M. J. Keeling, Proc. Natl. Acad. Sci. USA 99, 13330 (2002).

[35] T. Gross, Carlos J. Dommar DLima, and B. Blasius, Phys. Rev. Lett. 96, 208701 (2006).

[36] M. E. J. Newman, Networks an introduction (Oxford press) 2010.

[37] E. Volz, J. Math. Biol. 56, 293 (2008).

[38] E. M. Volz, J. C. Miller, A. Galvani, and L. A. Meyers, PLoS Comput. Biol. 7, e1002042 (2013).

[39] G. Caldarelli and A. Vespignani, Large scale structure and $d y$ namics of complex networks (world scientific publishing) 2003.

[40] M. E. J. Newman, Phys. Rev. E 66, 016128 (2002).

[41] R. Pastor-Satorras and A. Vespignani, Phys. Rev. E 65, 036104 (2002).

[42] Y. Chen, G Paul, S. Havlin, F. Liljeros, and H. E. Stanley, Phys. Rev. Lett. 101, 058701 (2008).

[43] R.-Q. Li, M. Tang, and P.-M. Hui, Acta. Phys. Sin. 62, 168903 (2013).

[44] K. Gong, M. Tang, P. M. Hui, H. F. Zhang, Y. Do, and Y. C. Lai, PloS ONE 8, e83489 (2013).

[45] H. Yang, M. Tang, and H. F. Zhang, New. J. Phys. 14, 123017 (2012).

[46] L. K. Gallos, R. Cohen, P. Argyrakis, A. Bunde, and S. Havlin, Phys. Rev. Lett. 94, 188701 (2005).

[47] X. Huang, J. Gao, S. V. Buldyrev, S. Havlin, and H. E. Stanley, 
Phys. Rev. E 83, 065101(R) (2011).

[48] J. Shao, S. V. Buldyrev, L. A. Braunstein, S. Havlin, and H. E. Stanley, Phys. Rev. E 80, 036105 (2009).

[49] M. E. J. Newman, S. H. Strogatz, and D. J. Watts. Phys. Rev. E 64, 026118 (2001).

[50] A. Clauset, C. R. Shalizi, and M. E. J. Newman, SIAM Rev. 51,
661 (2009).

[51] W. Wang, M. Tang, H. Yang, Y. Do, Y.-C. Lai, and G. W. Lee, Sci. Rep. 4, 5097 (2014).

[52] M. Barthélemy, A. Barrat, R. Pastor-Satorras, and A. Vespignani, Phys. Rev. Lett. 92, 178701 (2004). 